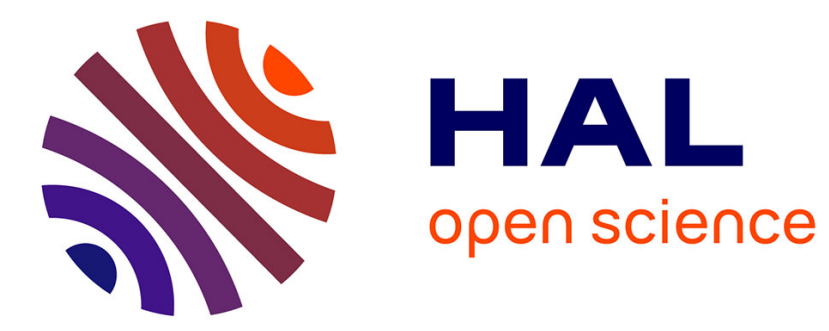

\title{
Violence et déchéance extrêmes
}

Delphine Scotto Di Vettimo

\section{To cite this version:}

Delphine Scotto Di Vettimo. Violence et déchéance extrêmes: Anne-Marie ou comment survivre à l'infamie. Les violences faites au corps des femmes, 2 (208), Erès, pp.33-44, 2015, DIALOGUE Familles \& Couples, 9782749247687 10.3917/dia.208.0033 . hal-01433548

\section{HAL Id: hal-01433548 https://hal-amu.archives-ouvertes.fr/hal-01433548}

Submitted on 17 Jan 2017

HAL is a multi-disciplinary open access archive for the deposit and dissemination of scientific research documents, whether they are published or not. The documents may come from teaching and research institutions in France or abroad, or from public or private research centers.
L'archive ouverte pluridisciplinaire HAL, est destinée au dépôt et à la diffusion de documents scientifiques de niveau recherche, publiés ou non, émanant des établissements d'enseignement et de recherche français ou étrangers, des laboratoires publics ou privés. 


\title{
Violence et déchéance extrêmes
}

\section{Delphine SCOTTO DI VETTIMO}

\author{
Psychologue clinicienne \\ Maître de Conférences en \\ Psychologie Clinique et Psychopathologie \\ Habilitation à Diriger des Recherches
}

\begin{abstract}
Aix-Marseille Université, Psychologie, LPCLS E.A 3278, 29 Avenue Robert Schuman,
13621 Aix-en-Provence Cedex 1

Adresse électronique : delphine.scotto-di-vettimo@univ-amu.fr

Téléphone (secrétariat) : 04-13-55-38-27

Téléphone (bureau) : 04-13-55-37-68
\end{abstract}




\section{Violence et déchéance extrêmes}

Delphine SCOTTO DI VETTIMO

\section{Résumé}

L'auteur explore, à partir d'une situation clinique, le champ des violences intrafamiliales extrêmes impliquant ici un inceste à la fois paternel, maternel et fraternel. C'est au décours de la maternité que ce passé traumatique (et non-dit) fut ravivé, nécessitant du sujet d'importants remaniements psychiques pour ne pas sombrer dans la dépression profonde. Le suivi clinique engagé montre les limites du processus thérapeutique, lorsque l'impensable et a fortiori l'irreprésentable provoquent une incapacité d'élaboration du trauma et son dépassement. Enfin, l'auteur propose d'éclairer les enjeux déterminants de la dynamique transférentielle et contre-transférentielle, pour le travail d'historicisation et d'inscription du trauma par le sujet, qui vont s'inaugurer par des modalités inédites de création subjective présageant d'un véritable processus de subjectivation.

\section{Mots-clés}

Déchéance ; inceste ; ontologie ; traumatisme ; violence.

\section{Violence and extreme decay}

\section{Summary}

This article explores the field of extreme intrafamily violences, from a clinical situation involving a paternal, maternal and fraternal incest. This traumatized and unspoken past was revived during the maternity and required vital psychic overhauls from the subject not to slide into deep depression. The clinical work initiated shows the limits of the therapeutic process when the unthinkable and a fortiori the unpresentable cause an incapacity of mental processing \& surpassing of the trauma. The author suggests providing an insight into the determinants of transferential and countertransferential dynamics for the work of historicisation and inscription of the trauma by the subject that will start by new modalities of subjective creation heralding a genuine process of subjectivation.

\section{Keywords}

Decay ; incest ; ontology ; trauma ; violence. 


\section{"Le dicible est préférable à l'indicible, La parole humaine au grognement animal». Primo Levi (Levi, 1984)}

«Durant les viols, ce n'est pas un homme mais une bête qui abusait de moi ». Il y a dans cet aveu saisissant d'Anne-Marie - difficilement soutenable pour la clinicienne que je suis - l'expression d'un espoir, aussi infime soit-il, que l'histoire de sa vie, marquée dans son enfance et son adolescence par un inceste familial d'une rare violence, ne soit plus le fait historique dramatique qui résumait et réduisait jusque-là - de façon implacable - sa vie. Une vie brisée.

À cette révélation - dans une formulation laconique effroyable - succèdera un effondrement corporel et physique de la patiente, mais aussi psychique...

Être psychologue, c'est précisément être confronté à de telles situations qui viennent interroger ce qui se manifeste dans un tel contexte. Il y faut entendre le souci d'être présent dans l'accueil d'une souffrance singulière privée de ses mots, en détresse pour dire l'indicible comme métaphore du ravage de la parole humaine et sa réduction violente au silence. L'impensable et a fortiori l'irreprésentable viennent pétrifier toute œuvre de parole et nous confrontent au vertige monstrueux du vide, à l'insupportable déshumanisation qui l'habite, à une désubjectivation qui installe les protagonistes dans une dimension de dépouillement, de dépeuplement et d'errance sans possibilité de restauration langagière, là où les mots deviennent dérisoires face ce qui relève de l'innommable.

Mais entre le silence muet de l'impartageable et la nécessité de partager, il y a le travail clinique, à l'épreuve. Dans cette perspective et ce sera ici le propos, je propose d'interroger - dans le champ des violences faites aux femmes et plus particulièrement à l'appui de l'histoire d'Anne-Marie - la question de la reconquête et/ou de la quête de soi comme ultime raison de vivre et de son corollaire - l'idéal esthétique - qui nous intéressera au titre de ce qu'il révèle ici de la position du sujet, confronté à une tragédie existentielle et subjective, véritable impasse de l'affirmation de l'être. Une impasse qui va trouver dans l'idéal esthétique son registre le plus singulier. Comme le plus inédit. 


\section{Un appel à l'aide}

Anne-Marie est une jeune femme de 33 ans dont la demande d'hospitalisation dans un service psychiatrique reposait sur un état dépressif récurrent, mais surtout sur une tentative de suicide par phlébotomie et ingestion médicamenteuse. La patiente décrivait alors un pessimisme, une incapacité à assumer ses responsabilités, une anxiété généralisée avec des plaintes somatiques multiples. Lors de l'entretien d'admission, elle évoquera également une violence contenue depuis plusieurs années et ravivée depuis sa maternité.

\section{$\underline{\text { Récits cliniques }}$}

Dès le début de la prise en charge, Anne-Marie avait exprimé des sentiments profonds de dévalorisation qui étaient à la source de ses idées suicidaires et qui révélaient surtout une grande fragilité narcissique. Très rapidement, elle avait évoqué l'inceste familial dont elle et ses sœurs avaient été victimes durant leur enfance : "J'ai eu une enfance plutôt noire avec des parents incestueux. À l'adolescence, je suis tombée enceinte de mon père...je me sens sale dans mon corps». Effectivement, le dossier de soins mentionnait trois tentatives de suicide par intoxication médicamenteuse à l'âge de douze, treize et quatorze ans, la dernière tentative - qui faisait suite à une Interruption Volontaire de Grossesse - ayant nécessité un long séjour dans un service de réanimation. Elle évoquera les soupçons des Services Sociaux mais n'avoir rien voulu révéler, de crainte de la rupture familiale brutale que sa dénonciation aurait provoquée. Curieusement, le discours d'Anne-Marie apparaissait dénué d'affects et d'émotions, en total décalage avec la gravité de son contenu. Elle rapportait son état dépressif à ces violences intrafamiliales, dépression réactivée depuis la naissance de son enfant car elle craignait de reproduire sur lui, ce qu'elle-même avait subi : "Tout allait bien jusqu'au moment de ma grossesse » dit-elle. Ce constat apparaissait comme faisant partie des effets de ravage de l'inceste, réminiscences d'un passé dont témoignaient aussi bien son impuissance que sa haine. Et si cette dernière constituait une tentative d'auto-guérison montrant ce que nous devons au réalisme de la haine, il y avait bien aussi dans ce viatique, le spectre fantomatique d'un abîme d'où les pensées surgissaient comme d'effroyables échos des violences physiques et sexuelles subies. Mathis, son fils, avait quatre ans; Anne-Marie relata une scène où elle lui mit une fessée car il était insolent : "J'ai peur de devenir comme mon père, je culpabilise d'avoir frappé mon fils ». Quatre ans, c'était l'âge où son propre père avait commencé à être violent à son égard. 
Pour ma part, je remarquais que ces propos dénotaient par moments une réelle difficulté à faire la part de sa propre identité, face à celle de son enfant. Par ailleurs, il convenait de souligner que dans la dynamique intersubjective, malgré les efforts de restitution d'un tissu associatif, la pensée s'enlisait. Les propos de la patiente portaient sur des évènements traumatiques de sa vie mais ne semblaient réveiller ni association ou réaction affective, l'inhibition venant réprimer toute expression d'affects. Dans le transfert, elle occupera de nombreux entretiens à relater ces relations empreintes de violence au sein de sa famille. À la tentative d'appropriation des faits par la patiente, se manifestait la tentative conjointe du clinicien de mettre en représentation ce qui était dit. Mais dans le contre-transfert, je me heurtais régulièrement à l'impossibilité d'appréhender l'inimaginable ou plutôt cette réalité crue dans toute son inhumanité. C'est comme si, dans la dynamique transféro-contre-transférentielle, nous étions prises dans le même gel des mouvements associatifs; à sa description itérative des faits se conjuguait mon incapacité à les penser. Que dire, si ce n'est que, d'une certaine manière, j'étais moi-même sidérée par l'horreur ? Ici, je souligne mon choix d'accorder une importance technique et théorique au transfert, qui sert d'instrument psychique (Fédida, 1992) pour la compréhension des expériences vécues par la patiente.

Durant les premières semaines d'hospitalisation, Anne-Marie pleura beaucoup durant les entretiens. Elle avait la conviction que sa vie entière était un échec généralisé et s'inscrivait dans une histoire «plutôt noire » dont elle reconnaissait tristement qu'elle était la sienne. Un jour, elle dit : «Je regrette de ne pas avoir dit «non » à mon père, je m'en veux, je me dégoûte moi-même». Le sentiment d'effroi qui accompagna cette affirmation avait pour corollaire l'horreur d'une passivité manifestée, qui l'avait laissée à chaque fois au bord du gouffre, rivée à un corps qui suscitait à présent le dégoût. Toutefois, le déchaînement ravageur de ce dégoût de soi, par le biais du travail clinique engagé, put laisser place, progressivement, à un retournement du dégoût en haine du père, comme processus de sauvegarde ultime...même si fragile...laissant toujours béante la blessure narcissique. Ce qui importait, avant tout, c'est qu'elle puisse enfin mettre en mots l'outrage extrême d'avoir été contrainte à des rapports sexuels avec son père, sa mère et ses sœurs : «Dire l'inceste, le révéler, c'est, pour la victime, trouver des repères à travers le langage, pouvoir symboliser ce qui ne pouvait pas l'être à l'origine (Razon, 1996, p. 223) soit mettre en évidence la part vivante du moi, son empreinte libidinale et les désirs qu'elle nourrit. 
Au-delà, Anne-Marie semblait rester captive de cet ensevelissement traumatique de l'être qui avait ravi son enfance et son adolescence et qui, faute de sépulture - et de symbolisation - la lestait dans un abîme sans fond, «[...] point ultime où le sens fuit de toute part et éperdument vers l'horizon du non-sens [...]»(Gori, 2009, pp. 38-39). Anne-Marie m'inquiétait car elle paraissait malgré tout figée dans une immobilité psychique qui cadenassait les frontières de son univers solitaire. D'autre part, cette inertie à penser et surtout élaborer en arrivait à contaminer nos rencontres : « Le contretransfert est une attitude interne ou une disposition ajustée qui se règle sur ce «senti inconscient. Il est donc acte d'intelligibilité subjective [...]»(Fédida, 1992, p. 198) dans le sens où il est la conscience du transfert. Ce qui s'apparentait ici à une impasse contre-transférentielle prendra plus tard la forme d'une co-construction psychique bénéfique. Le temps fut un allié décisif dans cette entreprise.

Un jour, elle confia : "Les images de l'inceste se déroulent comme un film permanent...je n'arrive plus à laisser le passé derrière moi...il m'obsède ». À cette évocation, elle se mit à sangloter et décréta, dans une formule lapidaire : "Je me sens coupable et honteuse de ce que j'ai fait». Anne-Marie tentait en vain d'effacer les souvenirs de son enfance qui prenaient figures d'obsessions : elle relata le début des violences physiques paternelles à l'âge de quatre ans et les premiers attouchements sexuels à l'âge de huit ans. Les révélations qui suivront dépassent toute possibilité de mise en représentation, tant elles mettent en relief l'horreur de la transgression maternelle et paternelle de la Loi de l'interdit de l'inceste : "Les attouchements sont devenus de plus en plus intimes...puis mon père a commencé à abuser de moi quotidiennement. Rapidement, il a demandé à ma mère et mes sœurs de participer. Puis il a décidé de nous filmer pour faire des films pornographiques ». Cet énoncé « forçait » littéralement l'oreille de la psychologue par sa monstruosité et surtout par la volonté totalitaire qu'il impliquait.

\section{Violence, démythification et perversion}

À la lumière des mythes fondateurs qui regorgent d'actes transgressifs où violence et sexualité sont explicitement mêlées, on sait que si la sexualité peut être fondatrice et structurante, alors quels mécanismes peuvent sous-tendre sa bascule tragique vers l'inceste ? Ici doit être évoquée - dans une métaphore explicite - la figure ravageante du père de la horde...père jouisseur dont le déchaînement pulsionnel et tyrannique se 
manifestait en un véritable pouvoir despotique de vie et de mort. Soit de terreur absolue. « Du père au pire » pour paraphraser la formule lacanienne, augure ici, à l'appui de Totem et Tabou (Freud, 1912) de cette clinique « du pire » où la consommation de ses propres enfants se constitue comme la transmutation du «repas totémique » au sens freudien, dans une entreprise de démythification du socle éthique par limitation des pulsions ainsi franchie. En d'autres termes prédominait, dans la constellation familiale, la toute-puissance de ce père totémique tel qu'il est décrit dans ce manifeste de l'anthroplogie psychanalytique, figure paternelle qui se situe à la fois du côté de la loi et du dérèglement de cette loi, à la fois du côté du désir et de la jouissance. Comme le souligne Jean Clavreul, « le pervers n'a pas réussi à éprouver sa solidité (de la loi) en la rattachant à son origine dans la différence des sexes et de l'interdit de l'inceste » (Clavreul, 1987, p. 199). Investi de l'illusion d'un pouvoir suprême, le père s'arrogeait un droit de vie et de mort sur sa famille, sa violence physique et verbale venant «bâillonner » toute tentative d'opposition et assujettir femme et enfants à sa barbarie.

Ce huis-clos effroyable durera des années, confirmant l'affirmation de Laure Razon selon laquelle « [...] l'arme la plus redoutable et la plus redoutée dont use l'abuseur est la menace, verbale ou physique »(Razon, 1996, p. 105). Enfin, l'autre forme de violence éprouvée par la patiente est liée à la mère - complice active - qui, dans un déni massif des faits, faisait directement écho à la violence du père puisque se soumettant à sa loi comme unique référent et n'attribuant aucune valeur à la norme sociale (Razon, 1996, p. 129). Anne-Marie se sentait torturée par cet inceste qui colonisait sa vie, dévastant peu à peu son monde intérieur et qui lui firent dire un jour : "J'ai été humiliée dans mon corps de petite fille et d'adolescente. Cette honte et cette salissure sont indélébiles ». Et elle s'effondra sur sa chaise. Ici, l'effondrement n'était pas à entendre du côté de son expression métaphorique classique, mais dans le sens d'un délitement $\mathrm{du}$ corps - «la souffrance détisse le corps » nous fait remarquer Pierre Fédida - d'un effacement en quelque sorte de l'apparence humaine. Cette observation clinique - versus manifestations corporelles et physiques - n'est pas sans rappeler une autre formule de Pierre Fédida, qui situe - chez les sujets qui ont vécu des situations extrêmes - un processus où « véritablement, d'un seul coup, se défait une expérience de l'humanité » (Fédida, 2007, p. 13) dans le sens où prévaut in fine le risque de perte de soi. Ici s'actualiserait en filigrane l'ultime espérance de retrouver une image humaine - 
ne serait-ce qu'à ses propres yeux - dans laquelle elle serait susceptible de se reconnaître.

\section{Survivance et honte, un destin commun}

Si je donnais ici une formulation résumée de ma perception, je dirais que dans les faits rapportés, il semble qu'Anne-Marie était en position d'objet littéralement « sadisé » par son père, dont les conduites pathologiques et les alcoolisations quotidiennes étaient vécues par la patiente comme la menace perpétuelle d'un risque d'anéantissement psychique et même physique

Elle dit s'être défendue de toutes ses forces, mais expliqua son impuissance à se protéger, se sentant sous son emprise et n'avoir pu faire face à ses avances perverses. Combien, en tant que clinicien, peut-on être sensible à ce moment qui peut se produire «[...] où le sujet se sent étrangement menacé d'anéantissement complet, total, parce que momentanément est perdu cet échange qui fait le tissu de l'humanité » (Fédida, 2007, p. 16) et où il ne peut pas se représenter cette déchéance...ne parvenant que, tout au plus, à la présentifier à l'autre comme ultime recours, faute de pouvoir soutenir la possibilité de la subjectiver. Anne-Marie en témoigna lorsqu'elle commença à fuir mon regard et dire ne plus supporter que je puisse la regarder. Ne pouvait-on pas émettre l'hypothèse que ce qui était là en jeu, c'était «[...] la représentabilité de ce qui est humain, ou de ce qui est inhumain, la question du représentable » (Fédida, 2007, p. 16) c'est-à-dire, en somme, ce qui fait le caractère irréductible de tout être humain ? Du fait même de la monstruosité de ce qu'elle avait subi, la patiente craignait de voir, dans mon regard, cette part monstrueuse d'elle-même, hors loi de l'interdit de l'inceste...

En outre, cette monstruosité se réactualisait à présent dans sa vie quotidienne et dans ses relations conjugales en particulier, dans le sens où elle «revivait» dans l'intimité de son couple la relation incestueuse : «Je ne supporte plus que mon mari me touche car je vois mon père à sa place» confia-t-elle à l'issue d'une permission. Quid de cette révélation stupéfiante, si ce n'est que la réactualisation des évènements traumatiques dans le transfert, avait pour corrélat une honte empreinte de sentiments incestueux qui venait à présent laminer et déliter sa vie sexuelle d'adulte ? Dans l'évocation de ce dédoublement d'images - «je vois mon père à la place de mon compagnon - qui s'imposaient à elle, Anne-Marie révélait que quelque chose ne pourrait jamais se transmettre, à savoir une expérience dont les mots s'avéraient impuissants à la soutenir, 
la représenter, l'expliquer. Et c'était bien de l'horreur du trauma dont il s'agissait. C'était cela aussi qui était informulable.

Enfin, cet envahissement par la honte comme indice d'une régression brutale aux temps originaires de l'existence, temps où l'objet laisse le sujet dans la déréliction, permettait de comprendre en quoi cette impuissance à avoir pu échapper à son bourreau, faisait écho à l'impuissance originaire de l'infans, aux expériences de passivation et de défaillance de la mère mais aussi à l'impuissance narcissique. Ainsi, ce vécu de déchéance évoqué maintes fois par la patiente, était à relier à l'expérience de passivation vécue comme un lâchage de la part de l'objet, véritable «perte de contenance devant le regard qui dénonce l'infamie » pour paraphraser Claude Janin (Janin, 2007, p. 101) et qui faisait dire à Anne-Marie que depuis, elle se vivait comme «un monstre » - à entendre comme expression métaphorique de cette expérience solitaire d'extrême passivation - et que la honte venait brutalement résumer comme expression de ce malaise. Dans la dynamique transféro-contre-transférentielle, la parole put se soutenir de la reconnaissance qu'elle trouva en son destinataire et du lien de confiance qui s'était ébauché. De surcroît, il convenait de noter que la mise en mots de la honte dans le travail clinique, avait nécessairement animé le lien transférentiel, c'était l'une des conditions qui ont permis de la travailler, de la reconnaître et de l'analyser.

Autant dire que la perception, par le sujet, à travers le regard de l'autre, de ses failles, de ses défaillances, est au premier plan de cet affect comme l'écrit Claude Barazer : «Il impose au psychisme des réponses urgentes de fuite, d'effacement, de dissimulation, comme en témoigne le lexique habituel : disparaître, rentrer sous terre » (Barazer, 2006, p. 61). D'où la difficulté à «l'approcher » dans le travail clinique. Et d'où l'évitement du regard manifesté par Anne-Marie à son évocation.

On retrouve chez Lévinas, dans son texte intitulé «De l'évasion» en 1935, cette prégnance de l'être qui affecte le sujet; cet affect ontologique est évoqué en termes de « sentiment aigu d'être rivé » (Lévinas, 1935, p. 95) à l'être même qui, dans sa crudité absolue, apparaît au sujet comme charge, poids ou encore «fardeau » (Heidegger, 1986, p. 178). Fardeau dont le sujet aspirera à s'affranchir, en vain. Car cette perfection de l'être qui vaut et qui pèse au sujet, provoque en somme la nécessité impérieuse d'évasion. Sinon...point de salut. En ce sens, le sujet honteux est celui qui doit assumer la responsabilité de son être...auquel il est inexorablement rivé. Ainsi, la honte se 
supporte d'une condamnation de l'Homme à assumer son être, à devoir endosser ce qui, dans le plus intime, lui est pourtant paradoxalement aussi le plus étranger.

Cette description ontologico-phénoménologique de l'expérience de honte apparaît ici comme la représentation allégorique et la figure de la nudité du corps et de la chair de l'Homme, ou encore comme exposition de la totalité de l'être...devenu objet sous le regard de l'autre étranger; étranger figuré par le clinicien, dans le transfert.

Pour résumer, les mots pour dire la honte ne doivent pas seulement être entendus dans ce qu'ils évoquent de souffrance, mais avant tout comme une parole «affectée » qui peut renvoyer à d'autres paroles, choses, évènements ; sa prise en compte conjointe dans le transfert et le contre-transfert, doit permettre au sujet de reconnaître la valeur subjective de cet affect...comme lien d'appartenance à l'espèce humaine, constitutive de l'altérité. Mais plus. Face à l'expérience solitaire d'extrême passivation inhérente à l'effraction traumatique que signe l'inceste, la honte comme véritable urgence éthique (Lévy, 2000, p. 121) chez Anne-Marie viendrait en souligner les éclats, comme expression de ce malaise de l'être, et dont la honte de vivre signe l'une des dialectiques possibles.

\section{Idéal esthétique et reconstruction narcissique, une dialectique circulaire}

On sait que face à la barbarie, il y a, au sens de Freud, cette volonté de la psyché de placer très haut ce besoin impérieux de causalité, cette faculté de la vie psychique qui consiste contre vents et marées à pouvoir transformer, dans sa réalité subjective, la réalité extérieure, c'est-à-dire la faculté de la psyché à traduire, transférer la force brutale du fait en significations, à transférer l'évènement lui-même, sa violence horssens, en lui donnant un statut psychique. Ce travail intrapsychique sera d'autant plus dynamique qu'il y a, du côté du sujet, une urgence à penser la réalité d'un désastre historique, familial, personnel (Lévy, 2000). Anne-Marie était précisément dans ce travail à la fois intra et intersubjectif. Dans la suite de cette prise de conscience, un changement fondamental s'opéra dans la relation transférentielle. Des possibles s'ouvrirent, augurant de mouvements psychiques dynamiques. Et non moins inattendus. C'est ainsi qu'au terme de l'hospitalisation qui aura duré quatre mois, Anne-Marie affirma ne pas souhaiter reprendre son activité professionnelle de secrétaire qu'elle décrivait pénible et sans intérêt. Elle émettra le projet d'un reclassement professionnel : «Je me suis renseignée pour suivre une école d'esthétique » dit-elle en esquissant un 
sourire. Je formulerai deux hypothèses sur le sens subjectif de ce choix : d'une part, le vécu narcissique de l'atteinte physique et psychique motiverait ce souhait de réorientation professionnelle : investir dans l'esthétique témoignerait du désir de trouver une image dans laquelle se reconnaître et s'aimer. En ce sens, devenir esthéticienne viserait implicitement à redéfinir les contours d'une identité, avec l'espoir que la nouvelle image socioprofessionnelle influencera l'identité subjective, l'image que la patiente a d'elle-même. Le régime alimentaire draconien qu'elle avait entrepris à cette même période, n'avait-il pas pour motif le désir explicite «de retrouver une image physique positive »? D'autre part, la préoccupation esthétique traduirait un contreinvestissement de la honte. Freud décrit, dans les Trois essais sur la théorie sexuelle - je le cite - les «forces psychiques» (Freud, 1905, p. 99) qui s'opposent à la pulsion sexuelle et qui, «telles des digues, resserreront son cours (le dégoût, la pudeur, les aspirations idéales esthétiques et morales) » c'est-à-dire qu'ici, honte et pudeur, honte et aspirations idéales esthétiques soulignent leurs liens sémantiques et psychiques, liens que fondent l'importance de la déclinaison des destins de la pulsion scopique, mais aussi le rôle de l'Autre ou celui de l'objet. À l'appui de cet argument théorique, ne pouvait-on pas émettre l'hypothèse qu'Anne-Marie tentait - ici une figure rhétorique prend toute sa portée - d'effacer la tache qu'elle incarnait à ses propres yeux et sur le corps social?

Ainsi, de cette tache inconvenante dans son histoire - la honte de l'inceste - où elle avait assisté, impuissante, à cette éclosion traumatique qui avait fissuré, fracturé puis fracassé tout son être, Anne-Marie essayait, à sa façon, par ce mécanisme psychique de conversion professionnelle, de se dégager de cette assignation de «victime d'inceste » qui ne faisait que la hanter, la lester et l'exproprier d'elle-même.

Cet espace psychique inédit augurait selon moi d'une possibilité d'appropriation subjective et symbolique. Et d'une reconquête de soi non dénuée d'une certaine créativité. Anne-Marie témoignait là d'une nouvelle spatialité interne et intime, comme d'une temporalité qui était désormais pourvue d'ancrage et d'horizon.

Nettoyer, effacer les traces...autant de signifiants éloquents susceptibles de triompher de la salissure. À la lumière de ce qui précède, si «la déchéance est une inclusion destitutive »(Barazer, 2006, p. 64) qui produit de la honte, honte d'être devenu déchet...honte de la déchéance infligée qui rejette le sujet dans une unicité indécente, alors peut-on avancer que pour Anne-Marie, il s'agissait en quelque sorte de prendre le 
contre-pied de cette assertion...soit de faire œuvre pour sortir de la honte, de la déchéance, en la transformant.

En ce sens et pour conclure, il convient de nous demander si l'assertion suivante : « $\mathrm{Ce}$ qui octroie à l'objet cette dimension de déchet nauséeux, c'est l'expérience d'un «être là » qui, ne renvoyant rien d' «autre » qu'à lui-même, est dépouillé de toute existence poétique outrepassant l'identité à soi-même » (Didier-Weill, 2010, p. 48) ne révèlerait pas, chez Anne-Marie, le prélude d'un travail d'élaboration psychique, prophétisant que l'affirmation qui précède n'était plus d'actualité, n'avait plus de raison d'être. AnneMarie était dans un processus de deuil bien entamé de mise en sépulture d'une enfance sacrifiée, pour sa survie subjective mais pas seulement. Ici, l'architecture d'une véritable opération psychique de subjectivation permettait d'en deviner le contour et d'en soutenir la gageure.

\section{EN CONCLUSION}

Avec l'inceste, nous sommes pris d'emblée dans une clinique de la honte, de l'humiliation et de la culpabilité dont les expressions paradigmatiques les plus manifestes sont l'attaque contre la filiation, la dignité, renvoyant chacun de nous au registre de l'innommable, de l'impensable et qui ont fait vivre Anne-Marie, durant toutes ces années, dans une familiarité horrible avec les réminiscences de l'inceste familial, dans ses aspects les plus crus comme les plus dévastateurs. L'espace clinique proposé, dont une des spécificités réside dans sa fonction de décalage, d'une écoute du « fait » humain, fait prévaloir un postulat fondamental qui est d'être toujours du côté du vivant : «[...] la vie, c'est la vie psychique » (Fédida, 2007, p. 251) et de favoriser la remise en mouvement, par la pensée puis le langage.

Cette épreuve plutôt fructueuse a fourni à Anne-Marie la possibilité d'élaborer autour de ce statut d'objet déchet qui l'avait engloutie outrageusement dans les ténèbres, là où la lumière du monde signifiant était devenue opaque, sédimentée. Un point lumineux semblait enfin advenir.

Je résumerai ici ce qui a constitué - dans le processus thérapeutique - le vecteur essentiel de la relation transférentielle et contre-transférentielle : «Cette capacité d'identification à l'autre, d'empathie, d'inférence, signifie que ce qui se passe est de l'ordre de l'humain. Car même si je ne l'ai pas vécu, je reconnais ce que l'autre a vécu, et en le reconnaissant comme étranger, je le reconnais comme semblable (Fédida, 2007, p. 37). 
Cela suppose que les affects, lorsqu'ils s'éprouvent ici et maintenant, appellent les images et les souvenirs (Chabert, 2003, p. 153) mais surtout les mots : des mots qui leur donnent droit d'asile voire de reconnaissance...et augurent d'un processus qui nous met à l'œuvre nous-mêmes.

\section{Références bibliographiques}

Barazer, C. 2006. «Quand le «propre» fait tache », Le Coq-Héron, 184, 57-67.

Chabert, C. 2003. Féminin mélancolique. Paris, P.U.F.

Clavreul, J. 1987. Le désir et la loi, Paris, Denoël.

Didier-Weill, A. 2010. Un mystère plus loin que l'inconscient, Paris, Flammarion.

Fédida, P. 2007. «L'oubli, l'effacement des traces, l'éradication subjective, la disparition », dans P. Fédida et al (sous la direction de), Humain/Déshumain. Pierre Fédida, la parole de l'œuvre, Paris, P.U.F., 11-124.

Fédida, P. 1992. Crise et contre-transfert, Paris, P.U.F.

Freud, S. 1912. Totem et Tabou, Paris, Payot, 1965.

Freud, S. 1905. Trois essais sur la théorie sexuelle, Paris, Gallimard, 1987.

Gori, R. 2009. «L'enfant mort des passions », dans J. André (sous la direction de), Folies de femmes, Paris, P.U.F., 27-46.

Heidegger, M. 1986. Etre et temps, Paris, Gallimard.

Janin, C. 2007. La honte, ses figures et ses destins, Paris, P.U.F.

Levi, P. 1984. À une heure incertaine, Paris, Gallimard, Collection « Arcades ».

Lévinas, E. 1935. De l'évasion, Paris, Fata Morgana, 1982.

Lévy, G. 2000. Au-delà du Malaise, Psychanalyse et barbaries, Toulouse, Érès.

Razon, L. 1996. Énigme de l'inceste, Paris, Denoël. 